\title{
Spatial Variability in Length Frequency Distribution and Growth of Shrimp (Pandalus borealis Kroyer 1838) in the Barents Sea
}

\author{
Michaela Aschan \\ Norwegian Institute of Fisheries and Aquaculture \\ N-9291 Tromsø, Norway
}

\begin{abstract}
Shrimp (Pandalus borealis) length frequencies from Barents Sea shrimp surveys were analysed to evaluate their usefulness in determining stock structure and optimising station groupings for assessment purposes. Four groups of stations were identified using cluster analysis on the Spearman coefficients correlating individual station length frequency distributions. The first group consisted mainly of small and some medium sized shrimp, the second mainly of medium and some small sized shrimp, the third mainly of medium and some large shrimp, and the fourth of stations having the largest shrimp. The spatial distribution of the groups was generally depth dependent, however in one year the group comprising the largest shrimp occurred in all depths, possibly due to migration or dispersion by currents. Modal analysis identified fifteen subareas with similar growth characteristics. The results are useful in optimising survey sampling design and improving input data for stock assessment models.
\end{abstract}

Key words: Barents Sea, growth, modal analysis, Pandalus borealis, shrimp, spatial distribution

\section{Introduction}

The shrimp (Pandalus borealis) fishery in the Barents Sea has no direct restrictions such as the Total Allowable Catches (TACs) used for most other North Atlantic shrimp stocks. Regulations in the Barents Sea include a minimum shrimp size $(15 \mathrm{~mm}$ carapace length), by-catch restrictions on young commercial fish, and limited entry. Reasons for not using TACs include: high natural mortality - shrimp consumption by cod (570 000 tons in 1994) (Bogstad and Mehl, 1997) by far exceeds shrimp catches (130000 tons in 1984, 30000 tons 1997) and, of particular relevance to the present study; lack of knowledge about stock structure and difficulties in defining suitable management units.

Kartavtsev et al. (1991; 1993) concluded from genetic and morphometric studies that there was no difference between samples collected within the Barents Sea. Comparative genetic allozyme and DNA analyses in the NE-Atlantic (Drengstig et al. 2000; Martinez et al., MS 1997) show obvious genetic gradients but no distinct sub-populations in the open Barents Sea.
Although management units have not been identified genetically, it may be possible to define them in other ways. The aim of this study was to describe the spatial and temporal variation in length frequency distributions (LFDs) and length-at-age of shrimp in the Barents Sea to determine if management units can be defined by similarities in life history characteristics.

\section{Materials and Methods}

\section{Study Area}

The northern part of the Barents Sea is covered by sea ice in winter, and is primarily under the influence of cold Arctic water masses, which flow into the area from the Northeast. Except for its easternmost part, the southern Barents Sea is ice-free all year round and is influenced by relatively warm Atlantic water masses (Fig. 1). Water temperatures in the Barents Sea can vary greatly depending on the amount of Atlantic water flowing into the area from the southwest (Aure et al., 1999). The periods $1987-89$ and $1994-98$ can roughly be characterized as cold and the periods 1990-93 and 1999-2000 as warm. 


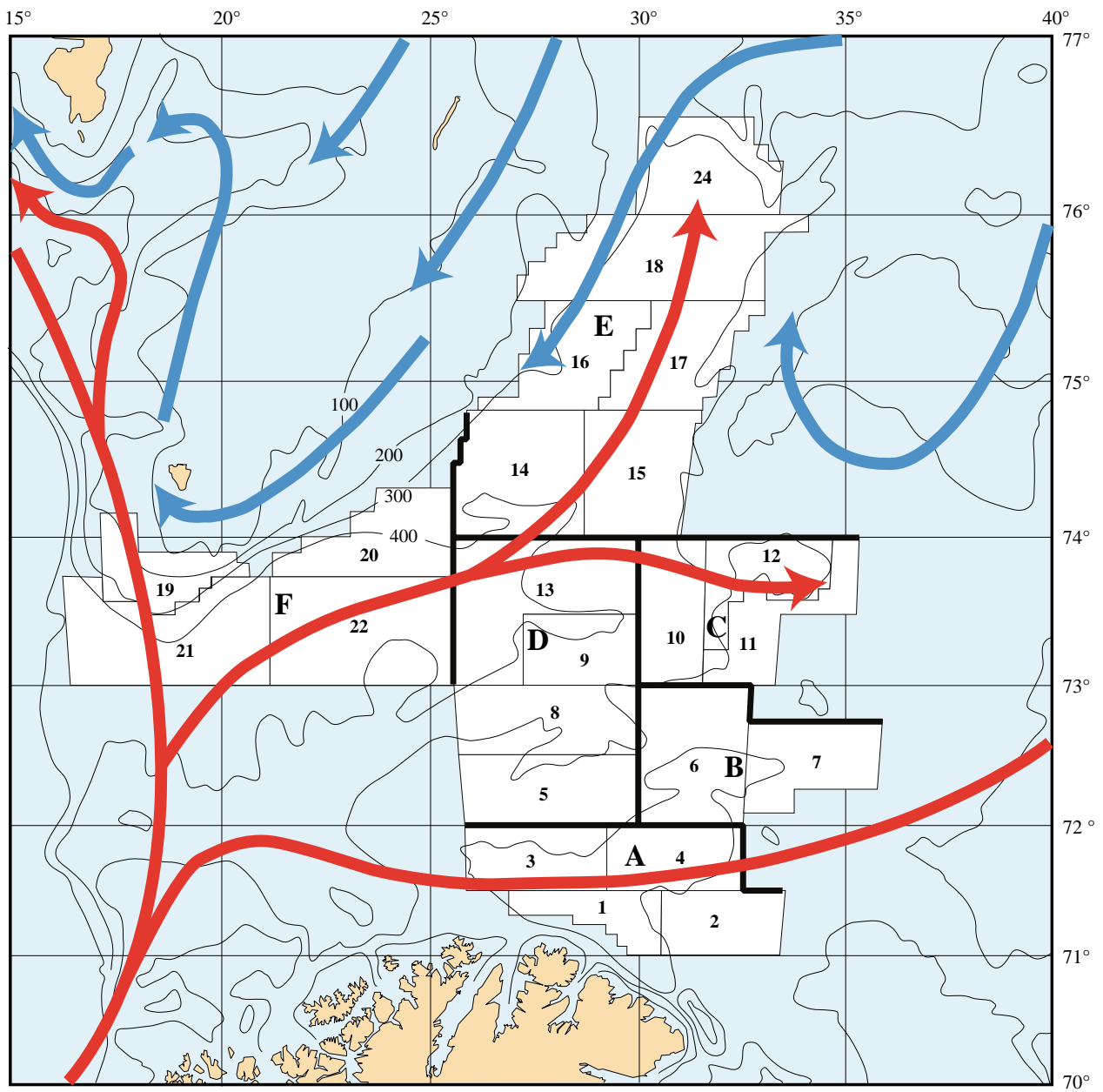

Fig. 1. A schematic description of the circulation of the Barents Sea. Arrows show the current of Atlantic water (red) and Arctic water (blue), and strata used in the Barents Sea for the shrimp surveys (1-22, 24). Main areas are (A) East Finnmark, (B) Tiddly Bank, (C) Thor Iversen Bank, (D) Bear IslandTrench, (E) Hopendeep and (F) Bear Island .

\section{Sampling and Laboratory Measurements}

Norway has conducted annual shrimp surveys in the Barents Sea during April-May since 1982. Aschan and Sunnanå (MS 1997) gave a detailed description of these surveys. The data for $1992-99$ are used in this study. A Campelen 1800 survey trawl was fished at stations selected systematically on a 20-30 nautical mile grid. The exception to this occurred in 1996 when the stations were spaced only 10 nautical miles apart in the northern region of the Barents Sea. The survey area is divided into six main areas including 23 strata (Fig. 1). Survey effort allocation is described and discussed by Harbitz et al. (1998).

Carapace lengths (CL) for length frequency information were measured from the posterior margin of the eyestalk to the posterior mid dorsal edge of the carapace. Approximately 300 individual shrimp from the catch at each trawl station were measured to the nearest $0.1 \mathrm{~mm}$ using digital callipers. The number of stations sampled and the number of shrimp measured annually are presented in Table 1 .

From 1992-95, an additional sub-sample (10 litres) was taken from the catch at each station and small shrimp (10-15 $\mathrm{mm} \mathrm{CL})$ were sorted out and measured to determine the presence or absence of small shrimp in the length frequency distribution. Since 1995 a quantitative method for measuring small shrimp was introduced when a small mesh $(0.8 \mathrm{~mm})$ nylon bag with a $1 \mathrm{~m}^{2}$ opening was attached to the under-belly of the survey trawl. This device samples 
TABLE 1. Number of strations and total number of individuals measured each year.

\begin{tabular}{lcccc}
\hline \hline Year & $\begin{array}{c}\text { Number of } \\
\text { stations } \\
\text { sampled }\end{array}$ & $\begin{array}{c}\text { Shrimp in } \\
\text { main sample } \\
\text { from cod end } \\
\text { (Numbers) }\end{array}$ & $\begin{array}{c}\text { Shrimp in } \\
\text { mesh bag } \\
\text { (Numbers) }\end{array}$ & $\begin{array}{c}\text { Small shrimp selected } \\
\text { from 10 litre sample } \\
\text { (Numbers) }\end{array}$ \\
\hline 1992 & 129 & 38290 & & 643 \\
1993 & 138 & 39202 & & 1138 \\
1994 & 107 & 32769 & 312 & 517 \\
1995 & 108 & 29712 & 1404 & \\
1996 & 144 & 42614 & 7688 & \\
1997 & 94 & 28445 & 855 & \\
1998 & 131 & 34742 & 737 & \\
1999 & 89 & 30201 & 3559 & \\
\hline
\end{tabular}

small shrimp (5-20 mm CL) efficiently, and provides an estimate of their density at each station. The samples of shrimp selected from the 10 litre sub-samples are not directly comparable with the mesh bag samples (Fig. 2).

\section{Data Analysis}

Spatial distribution. Statistical analyses were conducted using SYSTAT ${ }^{\circledR}$ (Wilkinson, 1990a) and SYGRAPH $^{\circledR}$ (Wilkinson, 1990b). For spatial analyses, only the main samples from the trawl cod end were used and additional samples for small shrimp were excluded. The length-classes $(0.1 \mathrm{~mm})$ were filtered with a weighted $0.5 \mathrm{~mm}$ moving average $\left(\left(\mathrm{n}_{1}+2 \mathrm{n}_{2}\right.\right.$ $\left.+3 n_{3}+2 n_{4}+n_{5}\right) / 9$ ) to smooth out measurement error. Samples were then sorted according to their similarity in LFDs with Spearman correlation. The resulting correlation matrix was used as input for a cluster analysis and for monotonic multidimensional scaling ordination (MDS). In the latter, data are analysed by

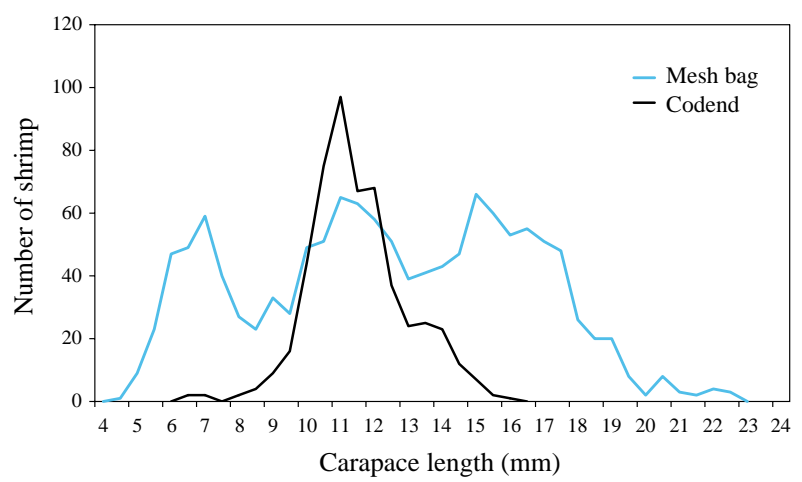

Fig. 2. Length frequency distribution of small shrimp selected from 10 litre samples from the codend, and shrimp sampled with a small mesh bag attached under the belly. Data from 1995 survey. similarities and split between matrices minimizing Kruskal stress in 2 dimensions. The cluster analysis gives distance values which indicate how well the sampling stations are correlated. Finally, values on the MDS axes (2-dimensions) are tested against environmental parameters such as depth, temperature, light, longitude and latitude. The analyses were run on LFD data from 1995-97 inclusive, but only the 1996 data are presented in detail to illustrate the procedure. All three years are included when evaluating the effect of environmental parameters on the spatial distribution of shrimp.

Growth. The Mix 3.0 modal analysis (Macdonald and Pitcher, 1979) of length frequencies was used to estimate the age composition of the survey samples. Small shrimp selected from the 10 litre sub-sample and/or the mesh bag sample were included and the LFDs were grouped into $0.5 \mathrm{~mm}$ bins. For 1993, 1995 and 1996 the Mix 3.0 analysis was conducted on the LFD for each station. Stations in the same main area having similar mean sizes-at-age were grouped by trial and error until 15 station groupings (subareas) were identified. Annual age frequency analyses were then run for each subarea for 1992-99 and growth patterns of individual year-classes within them were examined.

\section{Results}

Spatial distribution. The cluster analyses identified four distinct groups and seven single stations (here called outliers) at a distance of 0.15 (Fig. 3). As shown in Fig. 4, the first group consists of mainly small and some medium sized shrimp (labelled "small +"), the second of mainly medium and some small sized shrimp ("medium -"), the third of mainly medium and some large shrimp ("medium +"), and the fourth 
mainly of large shrimp ("large"). The monotonic multidimensional scaling ordination gave a stress of final configuration of 0.15 after five iterations, and a proportion of variance (RSQ) of 0.92. A twodimensional plot from the MDS for 1996 showed that the majority of variation between stations is explained by dimension 1 (Fig. 5).

Figure 6 shows that small and medium sized shrimp concentrated in the southern and eastern Barents Sea at a depth range of 200-350 m, while larger shrimp occurred in the northern and western Barents Sea at a depth range of 350-500 m. The four clusters were mainly distributed according to depth (Fig. 7). Correlations of dimensions 1 and 2 against environmental parameters for 1995-97 data show depth to be the most important variable (Table 2). For 1996 the correlation between dimension 1 and depth is even better $\left(r^{2}=0.46\right)$ when the fourth group (large shrimp) is excluded. The LFDs that comprise this group were not distributed according to depth as were other groups, but occurred at all depths (200-500 m) in 1996, possibly due to migration or dispersion by strong currents that year. Temperature appeared to be the second most important factor influencing the spatial distribution of shrimp. There was also a good

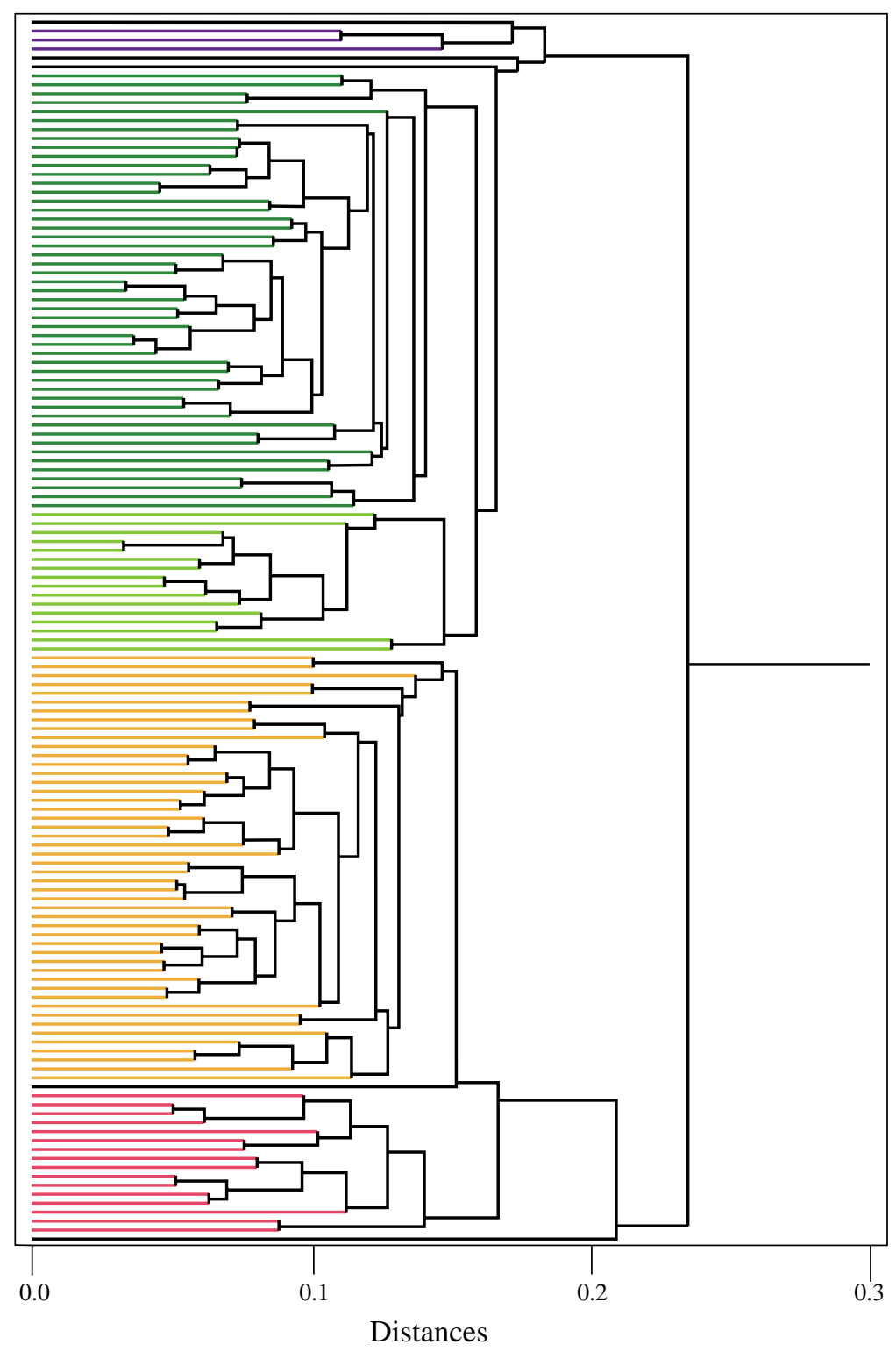

Fig. 3. Cluster analysis of distance between stations in the Barents Sea in 1996 according to length frequency distributions. 


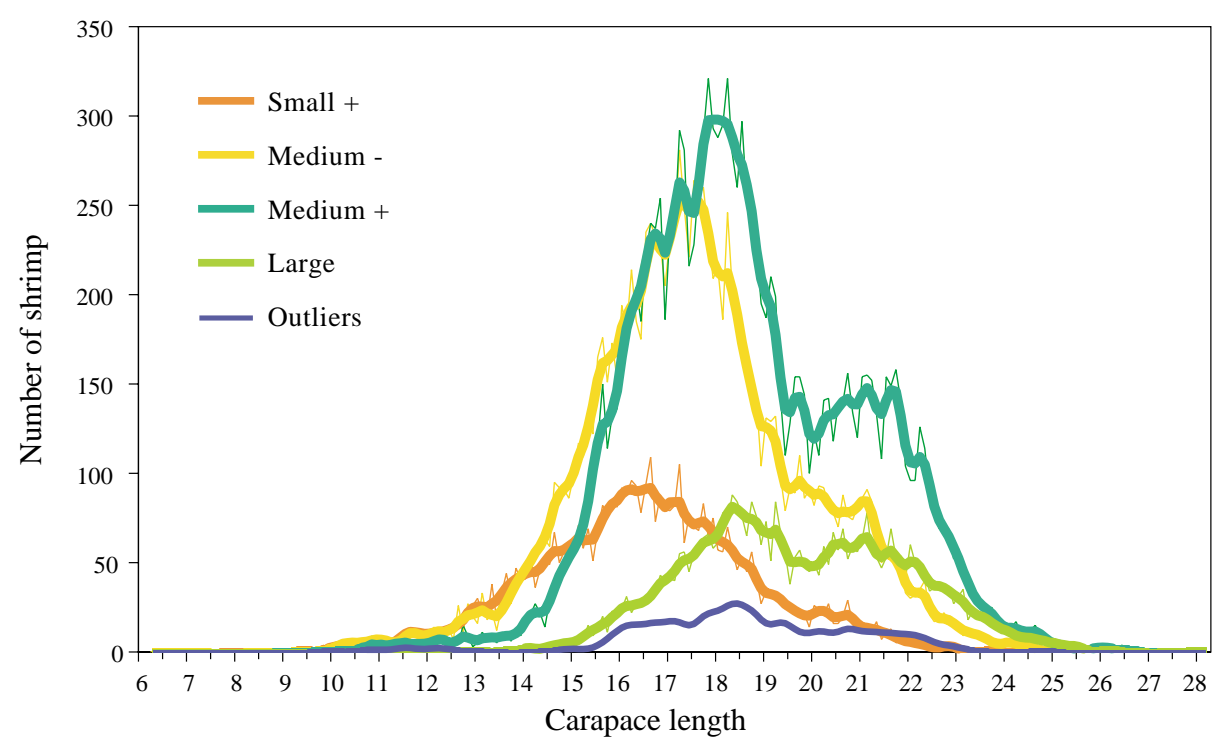

Fig. 4. Length frequency distributions representing four groups and the outliers ( 7 stations). Smoothed averages marked with thick lines and original data marked with thin lines.

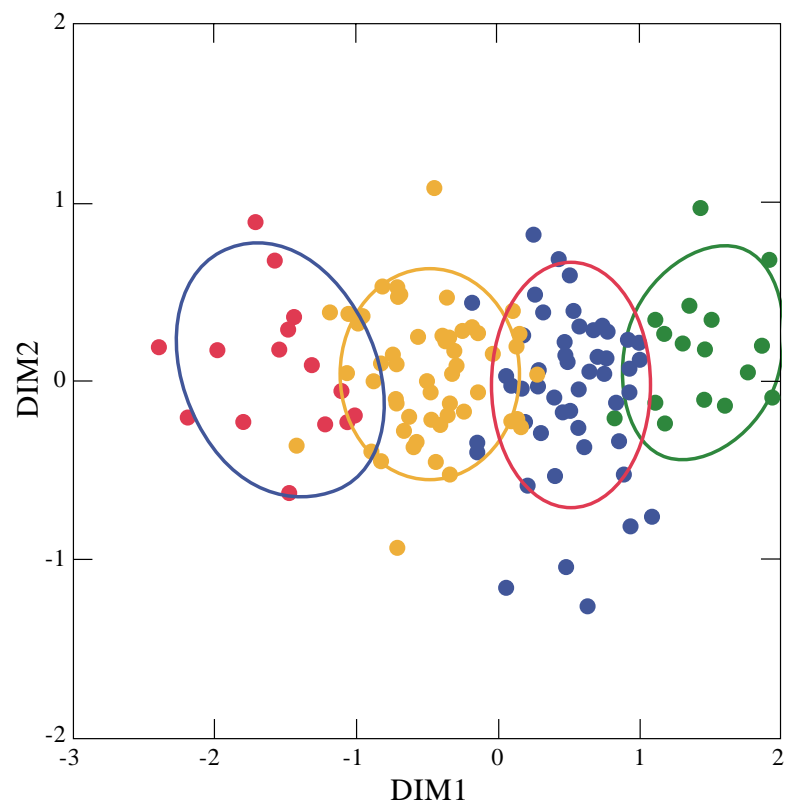

Fig. 5. Distribution of stations in the Barents Sea 1996 when analysed by monotonic multidimensional scaling ordination. The open circles from left to right corresponds to the four groups ranging from small to large indicated in Figure 4 and 6.

correlation with longitude and latitude, however these are not true environmental parameters and co-vary with other parameters such as temperature.

Growth. The cluster and MDS analyses described above, cluster analyses using transformed data and the Bray-Curtis index, and Principal Component Analysis were not useful for pooling samples having similar growth characteristics. Therefore neighbouring stations were pooled manually by trial and error i.e. if the LFDs had modes in the same position along the length axis.

Mean lengths, standard deviations (sd) and percent compositon for each year-class for 1992-99 showed no dramatic inter-annual differences (Table 3-10), although some years appeared to exhibit slower growth. The growth patterns of individual year-classes within the northern (strata 15, 16 and 17) and southern (strata 3 and 4) Barents Sea appeared to be fairly similar (Fig. 8). A comparison of the 1992 year-class clearly showed the difference in growth between the northern and southern areas (Fig. 9). The size of 0 -group shrimp was set to the carapace length of hatched larvae (zoea I) observed in the field. The growth curves appeared to become progressively steeper from the north to the south. Note that older age groups are difficult to distinguish due to the scarcity of large individuals in samples, particularly in strata 16-17 and 3-4, so the growth curves may give a false impression of decreased growth at these ages.

\section{Discussion}

The Barents Sea has relatively little depth variation, with no steep slopes, yet depth was found to be the main environmental factor explaining the spatial size distribution of shrimp. This is similar to 


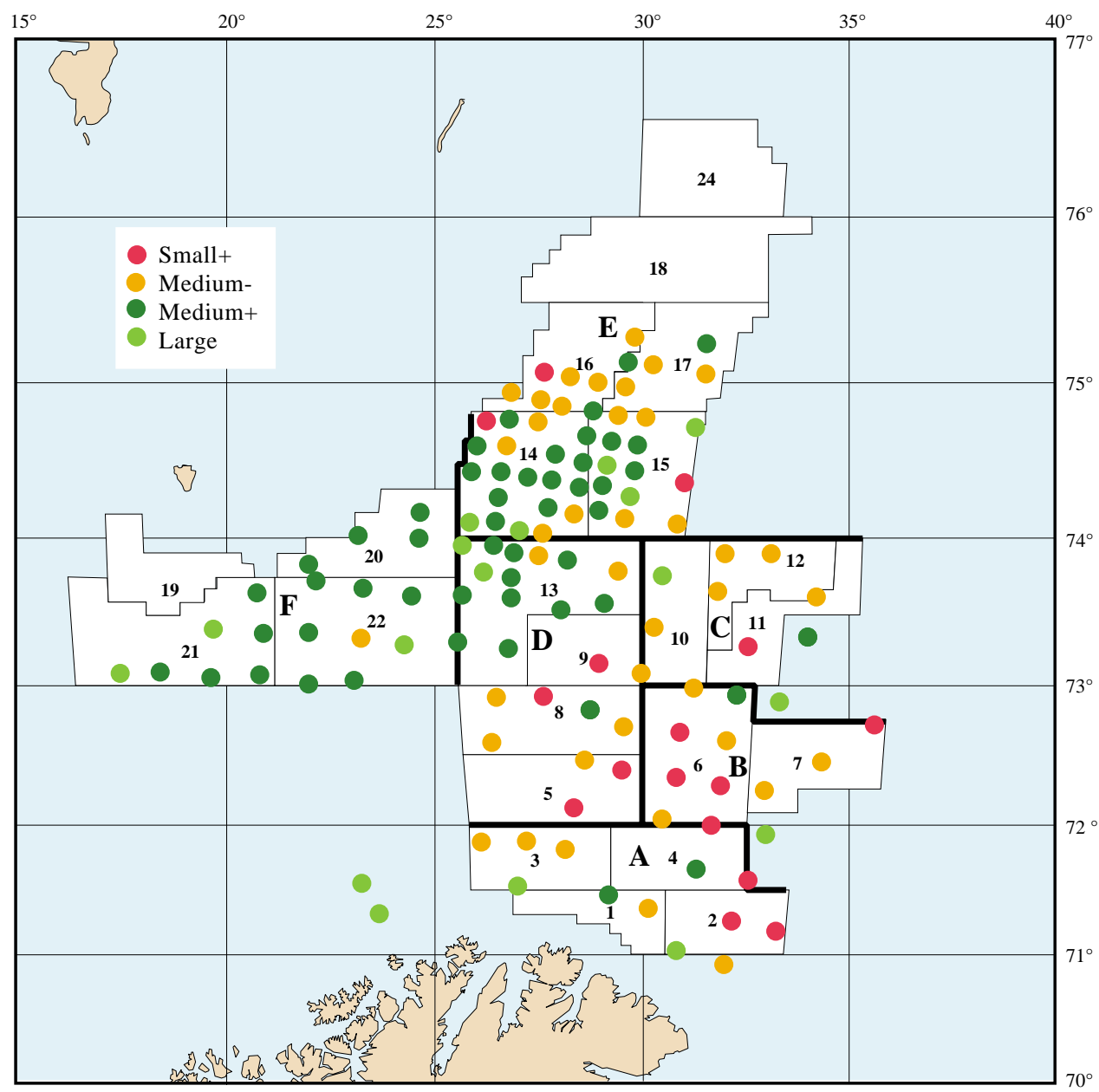

Fig. 6. Spatial distribution of four station groups characterised by length frequency distributions in the Barents Sea in 1996.

other areas such as the Gulf of St. Lawrence (Simard and Savard, 1990), Iceland (Skuladottir et al., 1991), Jan Mayen (Aschan et al., 1996) and Svalbard (Hansen and Aschan, 2000). Small and medium sized shrimp were common in shallow areas in the periphery of the present study area with a strong correlation between depth and size (Fig. 6 and Fig. 7). Medium and large shrimp are more common in the central parts of the study area (Fig. 1) and are associated with the Hopendeep (stratum 14) and the Bear Island Trench (strata 19-22). This spatial structuring of the population may result from better growth in the deeper areas or ontogenetic migration. The fourth group of samples with the largest shrimp that was not distributed according to depth in 1996 included many ovigerous females. These females, with eggs about to hatch, may have been migrating to shallower areas, and thus occurred at all depths. The migration of ovigerous females has previously been observed in the North
Atlantic (e.g. Shumway et al., MS 1985). Seasonal studies throughout the year are required to ellucidate shrimp migrations in the Barents Sea.

Since depth and temperature influence the size distribution of shrimp, it would appear to be appropriate to pool stations according to these parameters for stock assessment purposes. However, cluster analysis cannot distinguish the mean length of various modes and pools stations with substantially different modal peaks. Modal analysis fails when run on pooled data where the modal patterns are substantially different because the different modes blend and become more difficult to distinguish. For years when temperature gradients are narrow it may be possible to use larger pooled samples for the modal analysis. However, temperature usually varies greatly in the area, and is particularly influenced by the amount of Atlantic water flowing into the Barents Sea 
and by the position of the Polar front. These temporal and spatial variations in temperature result in local differences in growth. About 15 subareas appear to be appropriate in order to maintain the spatial resolution necessary to identify such differences.

Analysis of data from 1991-97 suggests that the shrimp in the south grow faster than those in the central and northern Barents Sea (Fig. 9). This agrees with earlier analysis conducted in the period 1979-81 (Teigsmark, 1983). Since growth rates vary

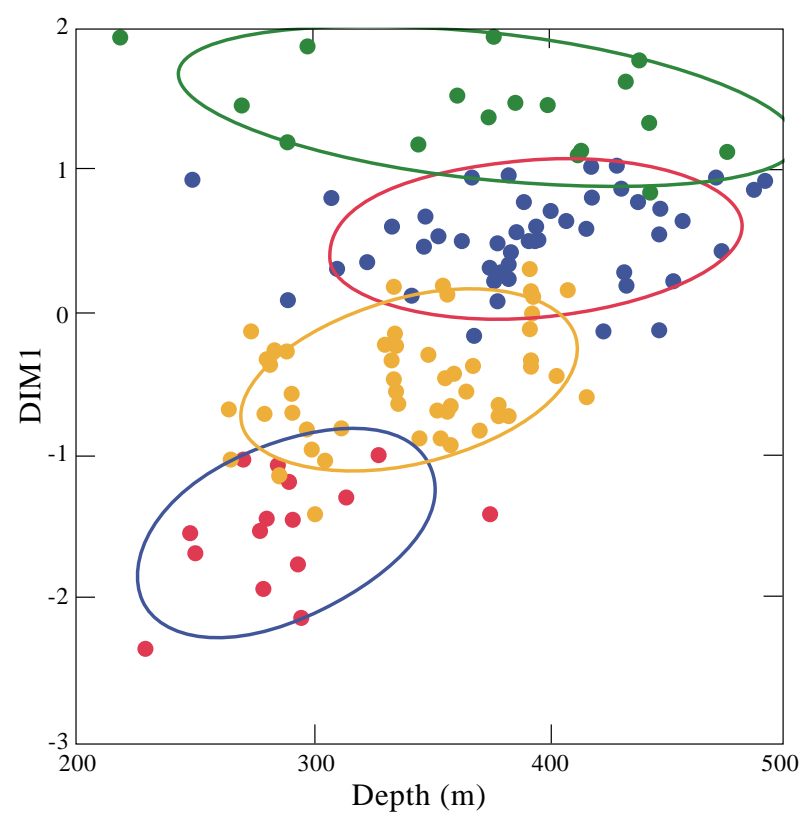

Fig. 7. Depth against value for dimension 1 from the monotonic multidimensional scaling ordination plotted for each station in the Barents Sea in 1996. The circles from bottom left to top right correspond to the four groups ranging from small to large indicated in Figure 4 and 6. between strata (Tables 3-10) it is desirable to define subareas with similar growth patterns and all modes present to determine cohort strengths in the area as a whole. Both small and large shrimp should be present in any defined subarea. Despite changes in size distribution from south to north and from west to east all four groups identified by the cluster analysis were present in the five main areas (A-E). However, the first group $($ small + ) was not present in area $F($ Fig. 6) and additional samples for these sizes in the shallow areas south of Bear Island would have improved the modal analyses in this area.

Nilssen and Hopkins (MS 1991) showed that, while significant latitudinal trends are present, the effects of specific environmental conditions (e.g. "warm" or "cold" current systems) at a given latitude, seasonal production cycles, and recent trends towards increased fishing effort on previously unexploited stocks are important factors modifying "latitudinal life cycle strategies" of this species. In the 1980s, when the water was "cold", the shrimp in the Barents Sea showed slower growth (Teigsmark, 1983) than in the warmer water period of the 1990s covered in the present study. Teigsmark (1983) identified three populations of Pandalus borealis in the Barents Sea that differed from each other by age at sex change and frequency of reproduction. The present study confirms that the variability of shrimp growth in the Barents Sea is high in space and time.

Berenboim and Lysy (1987) concluded that in the Barents Sea several shrimp sub-populations form a large super population through larval drift. This is supported by the recent genetic allozyme studies by Drengstig et al. (2000) and RAPD-analysis by Martinez et al. (MS 1997). These results suggest that future production and assessment models should consider the Barents Sea as one population. However,

TABLE 2. Squared multiple R for correlation between two dimensions (DIM) and enviromental parameters, latitude and longitude for 1995-97.

\begin{tabular}{lcccccc}
\hline \hline & & \multicolumn{2}{c}{1996} & & 1997 \\
Parameter & DIM 1 & DIM 2 & DIM 1 & DIM 2 & DIM 1 & DIM 2 \\
\hline Depth & .388 & .016 & .277 & .069 & .358 & .013 \\
Temperature & .043 & .056 & .000 & .025 & .100 & .263 \\
Light & .007 & .012 & .002 & .000 & .001 & .010 \\
ln light & .002 & .026 & .001 & .010 & .000 & .006 \\
Latitude & .015 & .164 & .012 & .102 & .349 & .006 \\
Longitude & .241 & .004 & .130 & .164 & .001 & .029 \\
Current & & & & & .001 & .029 \\
\hline
\end{tabular}


TABLE 3. Results of modal analysis (Mix 3.0) of length frequencies for each subarea or combination (stratum) in the Barents Sea in spring 1992, showing mean carapace lengths (CL in $\mathrm{mm}$ ) obtained for each year-class (YC) and proportion (given as percent) of YC in the total sample of shrimp (Number). Mix was run with the same standard deviation (sd) for all YC means.

\begin{tabular}{|c|c|c|c|c|c|c|c|c|c|c|c|c|c|c|c|c|}
\hline \multirow[b]{2}{*}{ Stratum } & \multirow[b]{2}{*}{$\mathrm{sd}$} & \multicolumn{2}{|c|}{ YC 91} & \multicolumn{2}{|c|}{ YC 90} & \multicolumn{2}{|c|}{ YC 89} & \multicolumn{2}{|c|}{ YC 88} & \multicolumn{2}{|c|}{ YC 87} & \multicolumn{2}{|c|}{ YC86 } & \multicolumn{2}{|c|}{ YC85 } & \multirow[b]{2}{*}{ Number } \\
\hline & & CL & Percent & $\mathrm{CL}$ & Percent & $\mathrm{CL}$ & Percent & $\mathrm{CL}$ & Percent & $\mathrm{CL}$ & Percent & $\mathrm{CL}$ & Percent & CL & Percent & \\
\hline \multicolumn{17}{|l|}{24} \\
\hline 22 & 1.18 & 6.46 & 1 & 11.85 & 3 & 16.68 & 16 & 19.01 & 38 & 21.33 & 42 & 24.04 & 1 & & & 4974 \\
\hline 21 & 1.12 & & & 12.90 & 2 & 17.25 & 17 & 19.72 & 41 & 21.65 & 39 & & & & & 2450 \\
\hline 19,20 & 1.31 & & & 11.58 & 5 & 16.12 & 24 & 19.15 & 37 & 21.37 & 35 & & & & & 2724 \\
\hline 18 & 1.02 & 7.37 & 2 & 12.39 & 19 & 16.30 & 42 & 8.77 & 14 & 21.52 & 23 & & & & & 1922 \\
\hline 16,17 & 0.97 & 7.55 & 1 & 11.87 & 25 & 15.70 & 32 & 18.05 & 13 & 20.70 & 11 & 22.01 & 16 & 24.58 & 2 & 3884 \\
\hline 15 & 1.11 & 7.40 & 1 & 12.59 & 6 & 16.59 & 28 & 18.86 & 25 & 21.94 & 35 & 24.78 & 4 & & & 2342 \\
\hline 14 & 1.65 & 6.84 & 1 & 13.30 & 7 & 17.26 & 57 & 21.70 & 36 & & & & & & & 2861 \\
\hline 9,13 & 1.10 & 7.48 & 1 & 11.94 & 4 & 15.81 & 10 & 18.45 & 38 & 21.21 & 42 & 23.10 & 6 & & & 3951 \\
\hline $10,11,12$ & 1.05 & 7.77 & 1 & & & 12.75 & 7 & 16.48 & 22 & 18.73 & 28 & 21.51 & 40 & 24.02 & 2 & 3011 \\
\hline 5,8 & 1.20 & 7.87 & 0 & 12.97 & 2 & 16.23 & 15 & 18.75 & 47 & 21.35 & 37 & & & & & 3774 \\
\hline 7 & 1.11 & 8.65 & 1 & 13.75 & 4 & 15.87 & 16 & 18.90 & 47 & 21.23 & 32 & & & & & 1943 \\
\hline 6 & 1.03 & 7.66 & 1 & 13.80 & 8 & 16.11 & 21 & 18.78 & 41 & 21.88 & 29 & 26.06 & 0.4 & & & 2026 \\
\hline 3,4 & 1.15 & 8.50 & 0 & & & 15.48 & 13 & 18.99 & 49 & 21.83 & 38 & & & 25.49 & 0.3 & 3139 \\
\hline 1,2 & 0.99 & & & 9.83 & 2 & 15.29 & 25 & 18.57 & 30 & 20.44 & 18 & 22.41 & 25 & & & 2311 \\
\hline
\end{tabular}

TABLE 4. Results of modal analysis (Mix 3.0) of length frequencies for each subarea or combination (stratum) in the Barents Sea in spring 1993, showing mean carapace lengths (CL in $\mathrm{mm}$ ) obtained for each year-class (YC) and proportion (given as percent) of YC in the total sample of shrimp (Number). Mix was run with the same standard deviation (sd) for all YC means. In parentheses are actual means when sample size was too small for Mix.

\begin{tabular}{|c|c|c|c|c|c|c|c|c|c|c|c|c|c|c|c|c|}
\hline \multirow[b]{2}{*}{ Stratum } & \multirow[b]{2}{*}{$\mathrm{Sd}$} & \multicolumn{2}{|c|}{ YC 92} & \multicolumn{2}{|c|}{ YC 91} & \multicolumn{2}{|c|}{ YC 90} & \multicolumn{2}{|c|}{ YC 89} & \multicolumn{2}{|c|}{ YC 88} & \multicolumn{2}{|c|}{ YC87 } & \multicolumn{2}{|c|}{ YC86 } & \multirow[b]{2}{*}{ Number } \\
\hline & & CL & Percent & $\mathrm{CL}$ & Percent & CL & Percent & CL & Percent & CL & Percent & CL & Percent & CL & Percent & \\
\hline 24 & & & & & & & & & & & & & & & & \\
\hline 22 & 1.09 & & & 11.22 & 2 & 15.73 & 18 & 18.02 & 42 & 21.20 & 36 & 23.69 & 1 & & & 2327 \\
\hline 21 & 1.12 & & & 12.07 & 2 & 16.19 & 15 & 18.10 & 35 & 21.13 & 38 & 23.02 & 10 & & & 3097 \\
\hline 19,20 & 1.27 & & & 11.27 & 1 & 16.13 & 37 & 18.61 & 30 & 21.33 & 30 & 24.27 & 2 & & & 1777 \\
\hline 18 & 1.13 & $(6.43)$ & & 10.53 & 11 & 14.73 & 37 & 17.29 & 34 & 20.68 & 13 & 23.13 & 5 & & & 1517 \\
\hline 16,17 & 1.03 & 6.39 & 1 & 11.28 & 4 & 15.27 & 35 & 17.90 & 35 & 21.01 & 17 & 23.00 & 6 & 25.39 & 2 & 1909 \\
\hline 15 & 1.13 & & & 11.78 & 2 & 15.77 & 26 & 18.23 & 38 & 21.53 & 26 & 24.11 & 7 & & & 1755 \\
\hline 14 & 1.05 & $(5.00)$ & & 11.87 & 2 & 15.74 & 32 & 18.15 & 44 & 21.16 & 17 & 23.53 & 5 & & & 1850 \\
\hline 9,13 & 1.27 & $(6.75)$ & & 10.92 & 1 & 15.29 & 14 & 18.04 & 51 & 21.27 & 33 & 24.60 & 1 & & & 2833 \\
\hline $10,11,12$ & 1.18 & $(6.50)$ & & 11.78 & 2 & 15.95 & 24 & 18.02 & 42 & 21.33 & 30 & 24.36 & 3 & & & 3861 \\
\hline 5,8 & 1.11 & $(7.35)$ & & 11.72 & 1 & 15.74 & 9 & 18.33 & 53 & 20.57 & 25 & 21.86 & 12 & & & 2342 \\
\hline 7 & 1.07 & 8.14 & 1 & 11.90 & 2 & 15.86 & 20 & 17.92 & 40 & 20.49 & 25 & 22.40 & 9 & & & 2337 \\
\hline 6 & 1.18 & 6.37 & 1 & 13.48 & 3 & 16.24 & 13 & 18.11 & 50 & 21.30 & 33 & 25.16 & 2 & & & 2342 \\
\hline 3,4 & 1.17 & 7.66 & 1 & 15.61 & 12 & 18.66 & 10 & 21.17 & 22 & 22.72 & 21 & & & & & 2817 \\
\hline 1,2 & 0.99 & 8.79 & 1 & 15.18 & 20 & 18.63 & 45 & 21.25 & 19 & 23.01 & 15 & & & & & 2667 \\
\hline
\end{tabular}


TABLE 5. Results of modal analysis (Mix 3.0) of length frequencies for each subarea or combination (stratum) in the Barents Sea in spring 1994, showing mean carapace lengths (CL in $\mathrm{mm}$ ) obtained for each year-class (YC) and proportion (given as percent) of YC in the total sample of shrimp (Number). Mix was run with the same standard deviation (sd) for all YC means. In parentheses are actual means when sample size was too small for Mix.

\begin{tabular}{|c|c|c|c|c|c|c|c|c|c|c|c|c|c|c|c|c|}
\hline \multirow[b]{2}{*}{ Stratum } & \multirow[b]{2}{*}{$\mathrm{Sd}$} & \multicolumn{2}{|c|}{ YC 93} & \multicolumn{2}{|c|}{ YC 92} & \multicolumn{2}{|c|}{ YC 91} & \multicolumn{2}{|c|}{ YC 90} & \multicolumn{2}{|c|}{ YC 89} & \multicolumn{2}{|c|}{ YC88 } & \multicolumn{2}{|c|}{ YC87 } & \multirow[b]{2}{*}{ Number } \\
\hline & & $\mathrm{CL}$ & Percent & $\mathrm{CL}$ & Percent & $\mathrm{CL}$ & Percent & $\mathrm{CL}$ & Percent & $\mathrm{CL}$ & Percent & $\mathrm{CL}$ & Percent & $\mathrm{CL}$ & Percent & \\
\hline 24 & 1.25 & & & 10.44 & 15 & 14.58 & 24 & 17.02 & 38 & 20.11 & 22 & 23.53 & 1 & & & 1327 \\
\hline 22 & 1.04 & 5.51 & 6 & 10.28 & 8 & 15.26 & 11 & 17.87 & 51 & 20.89 & 23 & 23.55 & 1 & & & 2393 \\
\hline 21 & 1.14 & 5.64 & 4 & 10.62 & 2 & 15.29 & 9 & 17.85 & 52 & 20.96 & 30 & 23.45 & 3 & & & 2002 \\
\hline 19,20 & 1.04 & & & 10.53 & 2 & 15.37 & 16 & 17.92 & 64 & 21.02 & 17 & 24.01 & 1 & & & 1298 \\
\hline 18 & 1.05 & 7.55 & 1 & 10.78 & 20 & 14.98 & 33 & 17.05 & 28 & 20.11 & 16 & 23.00 & 2 & & & 2168 \\
\hline 16,17 & 1.19 & & & 10.86 & 7 & 14.37 & 16 & 17.20 & 58 & 20.51 & 18 & 24.17 & 1 & & & 1424 \\
\hline 15 & 1.01 & & & 11.07 & 2 & 15.08 & 7 & 17.78 & 49 & 20.87 & 30 & 23.28 & 9 & 25.38 & 3 & 1790 \\
\hline 14 & 1.12 & & & 9.79 & 1 & 14.85 & 2 & 17.63 & 63 & 20.96 & 30 & 23.98 & 5 & & & 1867 \\
\hline 9,13 & 1.05 & & & 10.48 & 1 & 14.63 & 3 & 17.76 & 53 & 20.78 & 36 & 23.60 & 7 & & & 2912 \\
\hline $10,11,12$ & 1.15 & & & 11.56 & 2 & 14.72 & 8 & 17.36 & 40 & 20.58 & 41 & 23.11 & 9 & & & 2774 \\
\hline 5,8 & 1.13 & 8.18 & 1 & 10.06 & 1 & 15.39 & 15 & 17.82 & 45 & 20.68 & 33 & 22.91 & 5 & & & 2222 \\
\hline 7 & 0.96 & & & $(12.50)$ & & 14.67 & 22 & 17.46 & 41 & 20.29 & 28 & 22.31 & 9 & & & 623 \\
\hline 6 & 1.21 & & & 11.40 & 1 & 14.82 & 9 & 17.61 & 47 & 20.64 & 42 & 23.65 & 1 & & & 2632 \\
\hline 3,4 & 1.05 & 7.37 & 1 & 12.84 & 1 & 15.44 & 10 & 17.88 & 43 & 20.81 & 36 & 23.12 & 9 & & & 2780 \\
\hline 1,2 & 1.24 & 7.70 & 1 & & & 15.13 & 49 & 18.27 & 29 & 20.96 & 21 & & & 25.56 & 0.2 & 902 \\
\hline
\end{tabular}

TABLE 6. Results of modal analysis (Mix 3.0) of length frequencies for each subarea or combination (stratum) in the Barents Sea in spring 1995, showing mean carapace lengths $(\mathrm{CL}$ in $\mathrm{mm}$ ) obtained for each year-class (YC) and proportion (given as percent) of YC in the total sample of shrimp (Number). Mix was run with the same standard deviation (sd) for all YC means. In parentheses are actual means when sample size was too small for Mix.

\begin{tabular}{|c|c|c|c|c|c|c|c|c|c|c|c|c|c|c|}
\hline \multirow[b]{2}{*}{ Stratum } & \multirow[b]{2}{*}{$\mathrm{Sd}$} & \multicolumn{2}{|c|}{ YC 94} & \multicolumn{2}{|c|}{ YC 93} & \multicolumn{2}{|c|}{ YC 92} & \multicolumn{2}{|c|}{ YC 91} & \multicolumn{2}{|c|}{ YC 90} & \multicolumn{2}{|c|}{ YC89 } & \multirow[b]{2}{*}{ Number } \\
\hline & & $\mathrm{CL}$ & Percent & $\mathrm{CL}$ & Percent & $\mathrm{CL}$ & Percent & $\mathrm{CL}$ & Percent & $\mathrm{CL}$ & Percent & $\mathrm{CL}$ & Percent & \\
\hline 24 & 1.02 & 5.71 & 1 & 10.87 & 13 & 15.03 & 35 & 17.52 & 29 & 20.60 & 21 & 24.20 & 1 & 1691 \\
\hline 22 & 1.17 & 6.38 & 1 & 11.32 & 6 & 15.50 & 12 & 18.08 & 42 & 20.92 & 37 & 23.73 & 2 & 2353 \\
\hline 21 & 1.07 & 5.88 & 1 & 11.67 & 4 & 16.12 & 18 & 18.32 & 47 & 20.92 & 29 & 23.73 & 1 & 1481 \\
\hline 19,20 & 0.69 & 6.15 & 3 & 12.39 & 10 & 15.12 & 9 & 17.10 & 28 & 20.25 & 49 & 24.83 & 1 & 1393 \\
\hline 18 & 0.98 & 7.05 & 1 & 11.11 & 15 & 14.98 & 26 & 17.64 & 30 & 20.61 & 26 & 23.59 & 2 & 1970 \\
\hline 16,17 & 1.10 & 6.25 & 5 & 11.12 & 7 & 15.10 & 19 & 17.53 & 38 & 20.78 & 28 & 23.96 & 3 & 2163 \\
\hline 15 & 1.11 & 7.08 & 1 & 11.36 & 4 & 15.12 & 16 & 17.41 & 50 & 20.78 & 25 & 22.96 & 3 & 1134 \\
\hline 14 & 1.11 & 6.26 & 1 & 11.28 & 5 & 15.62 & 21 & 17.86 & 40 & 20.72 & 30 & 24.28 & 3 & 1411 \\
\hline 9,13 & 1.15 & 6.36 & 2 & 11.54 & 3 & 15.28 & 8 & 17.83 & 42 & 20.90 & 40 & 23.43 & 5 & 2585 \\
\hline $10,11,12$ & 1.09 & 6.95 & 1 & 11.36 & 5 & 15.13 & 14 & 16.94 & 44 & 20.45 & 35 & 23.18 & 1 & 2253 \\
\hline 5,8 & 1.40 & 7.68 & 3 & 12.61 & 8 & & & 17.20 & 74 & 20.14 & 15 & & & 1825 \\
\hline 7 & 1.20 & $(6.00)$ & & 10.01 & 3 & 13.28 & 8 & 16.25 & 50 & 20.29 & 34 & 22.93 & 5 & 919 \\
\hline 6 & 1.24 & 7.95 & 1 & 12.65 & 4 & 16.64 & 56 & 20.25 & 38 & 24.16 & 1 & & & 1553 \\
\hline 3,4 & 1.38 & 8.16 & 2 & 13.57 & 11 & 16.88 & 38 & 19.37 & 41 & 22.33 & 8 & & & 2391 \\
\hline 1,2 & 1.22 & 8.70 & 2 & 14.13 & 30 & 16.76 & 34 & 19.27 & 33 & 22.54 & 2 & & & 1461 \\
\hline
\end{tabular}


TABLE 7. Results of modal analysis (Mix 3.0) of length frequencies for each subarea or combination (stratum) in the Barents Sea in spring 1996, showing mean carapace lengths (CL in $\mathrm{mm}$ ) obtained for each year-class (YC) and proportion (given as percent) of YC in the total sample of shrimp (Number). Mix was run with the same standard deviation (sd) for all YC means. In parentheses are actual means when sample size was too small for Mix.

\begin{tabular}{|c|c|c|c|c|c|c|c|c|c|c|c|c|c|c|c|c|}
\hline \multirow[b]{2}{*}{ Stratum } & \multirow[b]{2}{*}{$\mathrm{Sd}$} & \multicolumn{2}{|c|}{ YC 95} & \multicolumn{2}{|c|}{ YC 94} & \multicolumn{2}{|c|}{ YC 93} & \multicolumn{2}{|c|}{ YC 92} & \multicolumn{2}{|c|}{ YC 91} & \multicolumn{2}{|c|}{ YC90 } & \multicolumn{2}{|c|}{ YC89 } & \multirow[b]{2}{*}{ Number } \\
\hline & & $\mathrm{CL}$ & Percent & $\overline{\mathrm{CL}}$ & Percent & $\mathrm{CL}$ & Percent & CL & Percent & CL & Percent & $\mathrm{CL}$ & Percent & $\mathrm{CL}$ & Percent & \\
\hline \multicolumn{17}{|l|}{24} \\
\hline 22 & 1.05 & & & 12.31 & 2 & 16.58 & 28 & 18.57 & 46 & 21.26 & 24 & 23.72 & 1 & & & 2301 \\
\hline 21 & 0.97 & & & 11.88 & 2 & 16.66 & 22 & 18.56 & 48 & 21.36 & 28 & 23.70 & 1 & & & 2032 \\
\hline $\begin{array}{l}19,20 \\
18\end{array}$ & 1.16 & & & 11.68 & 9 & 16.40 & 33 & 18.53 & 32 & 21.59 & 21 & & & 26.01 & 0.2 & 1812 \\
\hline 16,17 & 1.10 & $(6.50)$ & & 11.80 & 4 & 15.85 & 39 & 17.82 & 35 & 21.06 & 21 & 24.28 & 1 & & & 5431 \\
\hline 15 & 1.04 & & & 12.28 & 3 & 15.99 & 32 & 17.93 & 39 & 20.80 & 20 & 22.62 & 6 & $(26.00)$ & & 3876 \\
\hline 14 & 1.09 & $(8.00)$ & & 12.17 & 2 & 16.34 & 29 & 18.01 & 46 & 21.19 & 22 & 23.66 & 2 & & & 12652 \\
\hline 9,13 & 1.02 & $(7.50)$ & & 12.25 & 2 & 16.02 & 18 & 18.01 & 53 & 21.31 & 25 & 23.64 & 2 & & & 3623 \\
\hline $10,11,12$ & 1.06 & & & 11.92 & 2 & 15.15 & 17 & 17.28 & 54 & 20.47 & 25 & 22.82 & 3 & & & 2449 \\
\hline 5,8 & 1.26 & $(8.00)$ & & 12.36 & 2 & 15.12 & 17 & 17.37 & 69 & 20.37 & 11 & 21.86 & 12 & & & 2668 \\
\hline 7 & 1.13 & & & 11.38 & 3 & 14.44 & 17 & 17.06 & 65 & 20.30 & 15 & & & & & 1895 \\
\hline 6 & 1.16 & & & 12.19 & 4 & 14.71 & 13 & 17.02 & 69 & 20.30 & 13 & & & & & 1805 \\
\hline 3,4 & 1.22 & $(8.50)$ & & 13.83 & 9 & & & 17.42 & 55 & 20.74 & 27 & 24.27 & 7 & 27.87 & 2 & 2115 \\
\hline 1,2 & 1.17 & & & 14.68 & 36 & & & 17.90 & 44 & 20.70 & 16 & 22.80 & 5 & & & 1448 \\
\hline
\end{tabular}

TABLE 8. Results of modal analysis (Mix 3.0) of length frequencies for each subarea or combination (stratum) in the Barents Sea in spring 1997, showing mean carapace lengths (CL in $\mathrm{mm}$ ) obtained for each year-class (YC) and proportion (given as percent) of YC in the total sample of shrimp (Number). Mix was run with the same standard deviation (sd) for all YC means. In parentheses are actual means when sample size was too small for Mix.

\begin{tabular}{|c|c|c|c|c|c|c|c|c|c|c|c|c|c|c|c|c|}
\hline \multirow[t]{2}{*}{ Strata } & \multirow[b]{2}{*}{$\mathrm{Sd}$} & \multicolumn{2}{|c|}{ YC 96} & \multicolumn{2}{|c|}{ YC 95} & \multicolumn{2}{|c|}{ YC 94} & \multicolumn{2}{|c|}{ YC 93} & \multicolumn{2}{|c|}{ YC 92} & \multicolumn{2}{|c|}{ YC91 } & \multicolumn{2}{|c|}{ YC90 } & \multirow[b]{2}{*}{ Number } \\
\hline & & CL & Percent & $\overline{\mathrm{CL}}$ & Percent & CL & Percent & $\overline{C L}$ & Percent & $\overline{C L}$ & Percent & $\overline{C L}$ & Percent & $\mathrm{CL}$ & Percent & \\
\hline \multicolumn{17}{|c|}{24} \\
\hline 22 & 0.92 & & & 9.77 & 1 & 16.01 & 9 & 18.42 & 62 & 20.56 & 24 & 22.70 & 3 & & & 987 \\
\hline 21 & 0.96 & & & 10.27 & 10 & 15.92 & 7 & 18.32 & 51 & 20.92 & 33 & 22.97 & 7 & & & 1388 \\
\hline 19,20 & 1.11 & 4.07 & 1 & 10.48 & 1 & 15.21 & 7 & 17.81 & 45 & 21.38 & 45 & 24.19 & 2 & & & 995 \\
\hline 18 & 1.30 & $(5.16)$ & & 10.01 & 8 & 15.10 & 33 & 17.30 & 44 & 21.00 & 13 & 23.64 & 1 & & & 2137 \\
\hline 16,17 & 1.12 & 5.11 & 2 & 10.12 & 6 & 15.21 & 22 & 17.45 & 44 & 20.98 & 22 & 23.56 & 4 & & & 4616 \\
\hline 15 & 1.16 & 6.13 & 1 & 10.80 & 2 & 15.49 & 13 & 17.67 & 53 & 20.55 & 24 & 22.93 & 7 & 26.21 & 0.3 & 1955 \\
\hline 14 & 1.18 & 5.39 & 1 & 10.28 & 3 & 15.03 & 6 & 17.13 & 66 & 20.33 & 20 & 22.62 & 6 & & & 3506 \\
\hline 9,13 & 1.28 & 5.70 & 1 & 9.53 & 1 & 13.33 & 1 & 17.72 & 73 & 21.07 & 24 & & & & & 1431 \\
\hline $10,11,12$ & 1.20 & 4.99 & 1 & 10.03 & 4 & 14.50 & 11 & 17.09 & 51 & 20.17 & 32 & 23.53 & 1 & & & 2494 \\
\hline 5,8 & 1.06 & & & & & 15.04 & 6 & 17.48 & 17 & 20.03 & 15 & & & & & 1230 \\
\hline 7 & 1.11 & 6.49 & 1 & 10.30 & 3 & 14.47 & 12 & 17.14 & 56 & 19.94 & 26 & 22.8 & 1 & & & 974 \\
\hline 6 & 1.07 & 6.99 & 2 & 11.81 & 3 & 14.90 & 18 & 17.26 & 50 & 19.57 & 18 & 21.05 & 8 & & & 955 \\
\hline 3,4 & 1.13 & 7.15 & 0 & 11.70 & 1 & 15.15 & 6 & 17.81 & 65 & 20.33 & 25 & 22.8 & 4 & & & 2218 \\
\hline 1,2 & 0.99 & 7.01 & 1 & 9.50 & 0.4 & 14.71 & 7 & 18.04 & 62 & 20.23 & 25 & 22.57 & 5 & & & 1219 \\
\hline
\end{tabular}


TABLE 9. Results of modal analysis (Mix 3.0) of length frequencies for each subarea or combination (stratum) in the Barents Sea in spring 1998, showing mean carapace lengths (CL in $\mathrm{mm}$ ) obtained for each year-class (YC) and proportion (given as percent) of YC in the total sample of shrimp (Number). Mix was run with the same standard deviation (sd) for all YC means. In parentheses are actual means when sample size was too small for Mix. *indicates this stratum was combined with neighbouring area(s) to allow Mix analysis.

\begin{tabular}{|c|c|c|c|c|c|c|c|c|c|c|c|c|c|c|c|c|}
\hline \multirow[b]{2}{*}{ Statum } & \multirow[b]{2}{*}{$\mathrm{Sd}$} & \multicolumn{2}{|c|}{ YC 97} & \multicolumn{2}{|c|}{ YC 96} & \multicolumn{2}{|c|}{ YC 95} & \multicolumn{2}{|c|}{ YC 94} & \multicolumn{2}{|c|}{ YC 93} & \multicolumn{2}{|c|}{ YC92 } & \multicolumn{2}{|c|}{ YC91 } & \multirow[b]{2}{*}{ Number } \\
\hline & & $\mathrm{CL}$ & Percent & CL & Percent & $\mathrm{CL}$ & Percent & $\mathrm{CL}$ & Percent & $\mathrm{CL}$ & Percent & $\mathrm{CL}$ & Percent & $\mathrm{CL}$ & Percent & \\
\hline \multicolumn{17}{|l|}{24} \\
\hline 22 & 0.98 & $(5.00)$ & & & & 14.06 & 10 & 16.69 & 56 & 20.90 & 29 & 23.43 & 3 & & & 2460 \\
\hline 21 & 1.20 & 5.75 & 1 & 9.39 & 0.4 & 14.87 & 4 & 18.21 & 69 & 20.92 & 23 & 23.41 & 3 & & & 5049 \\
\hline 19,20 & 1.16 & $(5.00)$ & & $(10.00)$ & & 14.64 & 4 & 17.86 & 62 & 20.67 & 30 & 23.48 & 5 & & & 995 \\
\hline 18 & 0.87 & & & & & 14.98 & 46 & 16.96 & 34 & 18.78 & 10 & 21.26 & 10 & & & 334 \\
\hline 16,17 & 1.08 & 5.62 & 10 & 10.03 & 2 & 13.73 & 7 & 16.25 & 23 & 18.38 & 39 & 21.02 & 16 & 24.06 & 3 & 2549 \\
\hline 15 & 1.19 & 6.14 & 3 & 11.70 & 1 & 15.18 & 13 & 17.49 & 153 & 20.43 & 26 & 23.57 & 4 & $(26.00)$ & & 4761 \\
\hline 14 & 1.00 & 5.72 & 1 & 12.44 & 0 & 16.10 & 17 & 18.08 & 49 & 20.74 & 30 & 23.47 & 4 & & & 1926 \\
\hline 9,13 & 1.14 & $(6.00)$ & & 11.13 & 1 & 15.18 & 4 & 18.11 & 53 & 20.58 & 39 & 23.51 & 3 & & & 1431 \\
\hline $10,11,12$ & 1.20 & 6.20 & 4 & 11.72 & 17 & 15.31 & 12 & 17.60 & 43 & 20.53 & 36 & 23.22 & 3 & & & 2434 \\
\hline 5,8 & 1.11 & $(6.00)$ & & $(10.50)$ & & 14.53 & 7 & 17.07 & 72 & 19.50 & 19 & 21.37 & 2 & & & 1230 \\
\hline $7 *(6)$ & 1.33 & $(6.00)$ & & $(10.00)$ & & 14.50 & 5 & 16.78 & 44 & 19.69 & 48 & 22.61 & 4 & & & 1941 \\
\hline 6 & 1.03 & & & $(10.00)$ & & 13.75 & 2 & 16.21 & 21 & 18.02 & 53 & 20.16 & 19 & 21.42 & 6 & 904 \\
\hline $3,4 *(1,2)$ & 1.25 & & & 10.21 & 0.1 & 13.72 & 7 & 17.67 & 51 & 20.80 & 36 & 22.52 & 6 & & & 1219 \\
\hline 1,2 & 0.97 & & & 13.32 & 9 & 15.23 & 7 & 17.62 & 46 & 20.13 & 28 & 21.98 & 10 & 24.94 & 0.4 & 1857 \\
\hline
\end{tabular}

TABLE 10. Results of modal analysis (Mix 3.0) of length frequencies for each subarea or combination (stratum) in the Barents Sea in spring 1999, showing mean carapace lengths (CL in mm) obtained for each year-class (YC) and proportion (given as percent) of YC in the total sample of shrimp (Number). Mix was run with the same standard deviation (sd) for all YC means. In parentheses are actual means when sample size was too small for Mix. *indicates this stratum was combined with neighbouring area(s) to allow Mix analysis.

\begin{tabular}{|c|c|c|c|c|c|c|c|c|c|c|c|c|c|c|c|c|c|c|}
\hline \multirow[b]{2}{*}{ Statum } & \multirow[b]{2}{*}{$\mathrm{Sd}$} & \multicolumn{2}{|c|}{ YC 98} & \multicolumn{2}{|c|}{ YC 97} & \multicolumn{2}{|c|}{ YC 96} & \multicolumn{2}{|c|}{ YC 95} & \multicolumn{2}{|c|}{ YC 94} & \multicolumn{2}{|c|}{ YC93 } & \multicolumn{2}{|c|}{ YC92 } & \multicolumn{2}{|c|}{ YC91 } & \multirow[b]{2}{*}{ Number } \\
\hline & & $\mathrm{CL}$ & Percent & $\mathrm{CL}$ & Percent & $\mathrm{CL}$ & Percent & $\mathrm{CL}$ & Percent & $\mathrm{CL}$ & Percent & $\mathrm{CL}$ & Percent & $\mathrm{CL}$ & Precent & $\mathrm{CL}$ & Percent & \\
\hline 24 & 1.06 & 6.00 & 8 & 9.22 & 18 & 13.72 & 10 & 16.79 & 42 & 20.26 & 18 & 22.95 & 4 & & & & & 2407 \\
\hline 22 & 1.03 & 5.54 & 3 & 9.37 & 1 & & & 15.94 & 3 & 18.39 & 50 & 20.63 & 40 & 23.76 & 4 & & & 1286 \\
\hline 21 & 0.97 & $(5.00)$ & & $(9.50)$ & & & & $(16.50)$ & & 18.20 & 65 & 20.67 & 30 & 23.03 & 5 & & & 1580 \\
\hline 19,20 & 0.84 & $(5.50)$ & & & & 14.05 & 2 & 17.22 & 30 & 18.81 & 35 & 20.95 & 28 & 23.62 & 5 & 26.49 & 0.3 & 1300 \\
\hline 18 & 0.92 & 6.10 & 6 & 9.74 & 4 & 13.75 & 4 & 16.87 & 47 & 19.57 & 22 & 21.24 & 16 & 24.16 & 2 & & & 1888 \\
\hline 16,17 & 1.05 & 6.02 & 6 & 9.53 & 6 & 13.79 & 3 & 17.11 & 45 & 19.98 & 33 & 22.43 & 8 & 25.67 & 1 & & & 3104 \\
\hline 15 & 1.08 & 5.52 & 1 & 9.13 & 1 & 12.64 & 1 & 15.97 & 15 & 17.94 & 41 & 20.66 & 37 & & & & & 2239 \\
\hline 14 & 1.03 & 5.68 & 2 & 8.99 & 1 & 13.49 & 1 & 17.22 & 26 & 18.75 & 33 & 20.98 & 34 & 24.02 & 4 & & & 2254 \\
\hline 9,13 & 1.02 & 5.80 & 2 & 8.69 & 2 & 12.90 & 1 & 17.40 & 29 & 18.88 & 35 & 21.04 & 29 & 23.73 & 2 & & & 2339 \\
\hline $10,11,12$ & 1.35 & 5.31 & 3 & 9.72 & 3 & 13.40 & 2 & 17.49 & 58 & 20.69 & 25 & 24.23 & 1 & & & & & 4901 \\
\hline 5,8 & 1.05 & 5.72 & 1 & 10.27 & 1 & 15.05 & 2 & 18.29 & 66 & 20.79 & 29 & 23.09 & 2 & & & & & 1792 \\
\hline 7 & 1.31 & $(6.50)$ & & 10.50 & 2 & 13.31 & 3 & 17.38 & 60 & 20.31 & 31 & 22.80 & 4 & & & & & 2200 \\
\hline $6,7^{*}$ & 1.30 & 6.75 & 0.2 & 9.99 & 1 & 13.08 & 2 & 17.53 & 62 & 20.43 & 30 & 22.58 & 4 & & & & & 3999 \\
\hline 3,4 & 1.42 & 6.70 & 0.3 & 11.31 & 1 & 14.92 & 2 & 18.20 & 50 & 21.18 & 46 & & & & & & & 2063 \\
\hline 1,2 & 1.18 & 7.21 & 1 & 13.47 & 6 & 15.84 & 10 & 18.16 & 57 & 21.38 & 26 & 24.29 & 1 & & & & & 997 \\
\hline
\end{tabular}



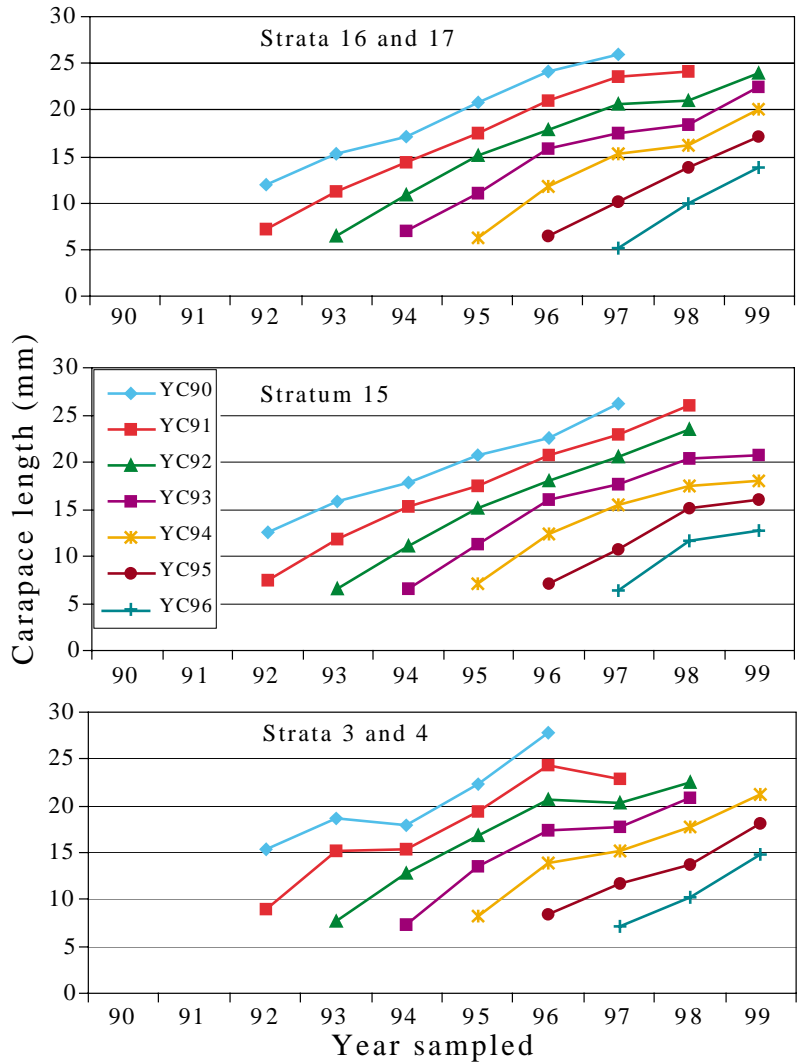

Fig. 8. Growth curves for 1990 to 1996 year-classes of shrimp in the northern Barents Sea (strata 16 and 17, and stratum 15) and southern Barents Sea (strata 3 and 4) in 1996.

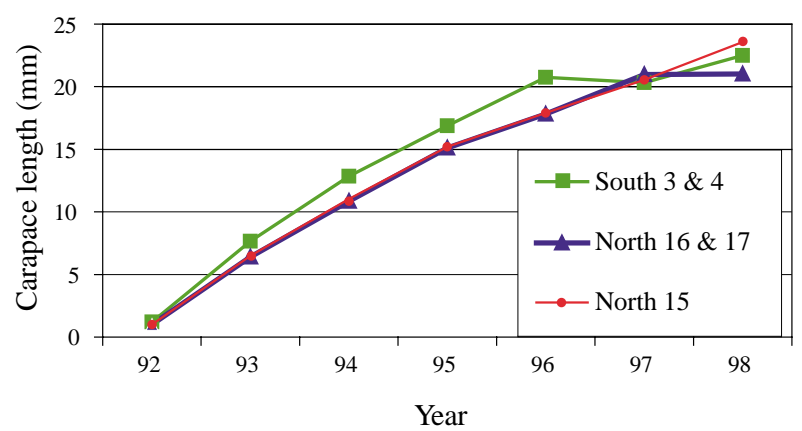

Fig. 9. Growth curves of the 1992 year-class shrimp in the northern Barents Sea (strata 16 and 17, and stratum 15 ) and in the southern Barents Sea (strata 3 and 4), based on data from surveys conducted in 199397.

such models usually require a fixed age for sex change and a constant growth rate. The present study indicates that the biological data should be organized on a detailed level e.g. 15 subareas for the Barents Sea. While separate analysis may be possible for each of these subareas, it would be logistically difficult, especially in providing adequate commercial samples. One solution could be to sum up the number of individuals in each year-class from each of the defined subareas and use these as input to an age based model. For a production model, the growth curve of the Hopendeep area may be used, as it represents a relatively large part (40\%) of the total stock biomass.

\section{Acknowledgements}

Marine research assistants Hege Øverbø Hansen, Karl-Erik Karlsen and Katrine Aase did a marvellous job by analysing many, many LFDs. Amanuenses Einar Nilssen has given me lots of good advice and support whenever needed. I thank the crew on board F/F "Jan Mayen" that showed patience during cruises and were of great help when solving practical problems on board. Research assistant Willy Richardsen took part in the last eight surveys and provided sampling expertise and knowledge about the survey trawl. Oddvar Dahl was very helpful with the graphics. Special thanks to Peter Koeller and Hege Øverbø Hansen who, with great patience, helped me with the final version of the paper.

\section{References}

ASCHAN, M., E. M. NILSSEN, L. H. OFSTAD, and S. TORHEIM. MS 1996. Catch statistics and life-history of shrimp, Pandalus borealis, in the Jan Mayen area. ICES C.M. Doc., No. K:11, 26 p.

ASCHAN, M., and K. SUNNANÅ. MS 1997. Evaluation of the Norwegian Shrimp Surveys conducted in the Barents Sea and the Svalbard area 1980-1997. ICES C.M.Doc., No.Y:07, 24 p.

AURE, J. Editor. 1999. Havets Miljø. 1999. Fisken Hav. Scernr., 2, $104 \mathrm{p}$.

BERENBOIM, B. I., and A. Y. LYSY. 1987. Effects of oceanographic factors on populational structure of shrimp (Pandalus borealis Krøyer) in the Barentas Sea and Spitsbergen area. In: The effect of oceanographic conditions on distribution and population dynamics of commercial fish stocks. Proceedings of the third Soviet-Norwegian Symposium, Murmansk, 26-28 May 1986. Ed: Harlad Loeng. Institute of Marine Research, Bergen, p. 243-250.

BOGSTAD, B., and S. MEHL. 1997. Interactions between Cod (Gadus morhua) and its Prey Species in the Barents Sea. Forage Fishes in Marine Ecosystems. Proceedings of the International Symposium on the Role of Forage Fishes in Marine Ecosystems. Alaska Sea Grant College Program Report No 97-01: 591-615. University of Alaska Fairbanks.

DRENGSTIG, A., S. E. FEVOLDEN, P. E. GALAND, and M. M. ASCHAN. 2000. Population structuring of the 
deep sea shrimp (Pandalus borealis) in the NE Atlantic based on allozymic differentiation. Aquat. Living Resour. 13: 1-9.

HARBITZ, A., M. ASCHAN, and K. SUNNANÅ. 1998. Optimum stratified sampling design for biomass estimates in large area trawl surveys - exemplified by shrimp surveys in the Barents Sea. Fish. Res., 37: 107113.

HANSEN, H.Ø., and M. ASCHAN. 2000. Growth, size and age at maturity of shrimp Pandalus borealis at Svalbard. J. Northw. Atl. Fish. Sci., 27: 83-91 (this volume).

KARTAVTSEV, Y. P, B. I. BERENBOIM, and K. I. ZUGOVSKY. 1991. Population genetic differentiation of the pink shrimp Pandalus borealis Kröyer, 1838, from the Barents and Bering Seas. J. Shellfish Res., 10: $333-339$.

KARTAVTSEV, Y. P., K. I. ZUGOVSKY, and Z. M. FEDINA. 1993. Spatial structure of pink shrimp Pandalus borealis Kröyer, 1838, From the Far-eastern Seas as provided by methods of population genetics and morphometrics. J. Shellfish Res., 12: 81-87.

MACDONALD, P. D. M., and T. J. PITCHER. 1979. Age groups from size-frequency data: a versatile and efficient method of analysing distribution mixtures. $J$. Fish. Res. Board Can., 36: 987-1001.

MARTINEZ, I., T. O. SKJELDAL, and S. M. ALJANABI. MS 1997. Genetic structuring of Pandalus borealis in the NE- Atlantic. II. RAPD analysis. ICES C.M. Doc.,
No. T:24, 14 p.

NILSSEN, E. M., and C. C. E. HOPKINS. MS 1991. Population parameters and life histories of the deepwater prawn Pandalus borealis from different regions. ICES C.M. Doc., No. K:2, 20 p.

SHUMWAY, S. E., H. C. PERKINS, D. F. SCHICK, and P. STICKNEY. MS 1985. Synopsis of biological data on the pink shrimp, Pandalus borealis Kröyer, 1838. U.S. Dept. of Comm. NOAA Tech. Rep. NMFS Doc., No. 30, $57 \mathrm{p}$.

SIMARD, Y., and L. SAVARD. 1990. Variability, spatial patterns and scales of similarity in size-frequency distributions of the northern shrimp (Pandalus borealis) and its migrations in the Gulf of St. Lawrence. Can. J. Fish. Aquat. Sci., 47: 794-804.

SKÚLADÓTTIR, U., J. PÁLSSON, G. S. BRAGASON, and S. BRYNJÓLFSSON. MS 1991. The variation in size and age at change of sex, maximum length and length of ovigerous periods of the shrimp, Pandalus borealis, at different temperatures in Icelandic waters. ICES C.M. Doc., No. K:5. 16 p.

TEIGSMARK, G. 1983. Populations of the deep-sea shrimp (Pandalus borealis Krøyer) in the Barents Sea. Fisk. Dir. Skr. Ser. HavUnders., 17: 377-430.

WILKINSON, L. 1990a. SYSTAT: the system of statistics. Systat Inc., Evanston IL.

1990b. SYGRAPH. The system of graphics. Systat Inc., Evanston IL. 
